\title{
Quantitative Stress Analysis Using Heart Rate Variability
}

\author{
Sayaka UENO ${ }^{\mathrm{a},{ }^{*}, \text { Runhe HUANG }}{ }^{\mathrm{b}}$, Satoru FUJITA ${ }^{\mathrm{b}}$ \\ ${ }^{a}$ Graduate School of CIS, Hosei University, 3-7-2 Kajino-cho, Koganei, Tokyo 184-8584 Japan \\ ${ }^{b}$ Faculty of CIS, Hosei University, 3-7-2 Kajino-cho, Koganei, Tokyo 184-8584 Japan \\ *Corresponding author: sayaka.ueno.5k@stu.hosei.ac.jp
}

\begin{abstract}
Several types of stress affect our lives, which can be attributed to the complexity of our current social structures and existence of advanced technologies such as the Internet. These stresses sometimes result in ill health. Therefore, awareness of our stress conditions is beneficial in reducing our stress levels and controlling our health by objectively evaluating and recognizing our stress conditions. This study proposes a quantitative stress index using the $\mathrm{R}-\mathrm{R}$ interval (RRI) of the electrocardiogram. This index contributes toward the reduction of the effects of variable ranges in heart rates. Our experiments showed that both RRI values and their dispersion became smaller and larger when we feel stressed and relaxed, respectively. This new stress index can be calculated using four steps: (1) RRIs are divided into small windows; (2) a change in RRI per beat is calculated in each window; (3) standard deviations of the changes in each window are calculated; and (4) standard deviations in each window are divided by the average of the RRIs in each window to reduce the effects of the RRI levels. Herein, these values are used to define a new stress index. We conducted two types of experiments namely, physical and mental stress to the body. In the case of physical stress, $t$-test results for the new index showed that the difference in the new index values between stressed and relaxed situations was statistically significant.
\end{abstract}

Keywords: stress index, R-R interval, heart rate variability, Poincare plot.

\section{Introduction}

Recently, the level of stress in peoples' lives has increased, and it has become difficult to spend a day or work without stress. Stress is a biological response to burdens from physical or mental stimuli. It is a symptom that should not be ignored because excessive stress results in a disordered life and disease. Therefore, we need to control and reduce our stress levels to maintain our health conditions, although we seldom observe and evaluate our stress levels objectively. Several studies analyze stress objectively by measuring some physical parameters observed in our bodies and autonomic nervous system. For example, the autonomic nervous system is noninvasively measurable using relatively low-cost medical equipment. Heart rate variability (HRV) is one of the most frequently used biological data in the analysis of the autonomic nervous system because the static balance of the autonomic nervous activity reflects in the heart rate. However, the index of the existing method does not work well for various stress situations. This study proposes a method to evaluate the stress value quantitatively using a new HRV index and analyze the HRV using time domain indicator. This study aims to create an index that is independent of heart rate interval levels.

\section{Related Work}

\subsection{R-R Intervals of Electrocardiogram}

An electrocardiogram is a graph of cardiac potentials of the human heart. Given that the human heart continuously works to provide blood to the whole body, the cardiac potentials need to activate the cardiac muscle cyclically. Therefore, cardiac potentials are shaped as cyclic waves. The highest peak of the wave in a cycle is called $\mathrm{R}$-wave, which is usually sharp and is easily captured. Thus, the interval of two consecutive R-waves, which is called R$\mathrm{R}$ interval or simply RRI, is measured as an inverse of the heart rate. If the human heart works at a completely regular rate, RRIs are stable and indifferent. However, the actual RRIs have fluctuation. This fluctuation can be attributed to many causes, including stress. 
The fluctuation also causes a cyclic change. The low and high frequency of the fluctuation ranging $0.05-0.15 \mathrm{~Hz}$ and $0.15-0.40 \mathrm{~Hz}$ is the LF and HF part, respectively. The HF fluctuation mainly comes from the parasympathetic nervous system that controls the respiratory organs, whereas LF fluctuation comes from both parasympathetic and sympathetic nervous systems. When we feel stressed, the sympathetic nervous system is activated, and the parasympathetic nervous system is deactivated. When we feel relaxed, the systems are reversed. Then, the ratio of LF and $\mathrm{HF}, \mathrm{LF} / \mathrm{HF}$, can indicate the human stress conditions. Generally, we are stressed when LF/HF is higher; otherwise, we are relaxed. LF/HF is widely used as an index of stress but the resolution of the low frequency domain in the RRI fluctuation is sometimes poor. Therefore, LF/HF should only be a considerable reference of the stress conditions. As another research, Vuksanović ${ }^{(1)}$ found that increase of heart rate in the case of mental stress could be achieved through the decreased and increased modulation in HF. This indicated that HF was more important than the LF to estimate the stress situation.

\subsection{Hitoe: a Platform for Electrocardiogram}

Hitoe $^{(2)}$ is a functional material sold in a public market and is also a platform to measure semimedical health data. It provides a cloth electrode material for measuring biomedical signals, such as electrocardiogram and myoelectricity, and is combined with an inner shirt to allow the attachment of this material to human skin easily. This facilitates the measurement of heart rate fluctuations and electrocardiograms for a long period of time. It has an improved adhesion to the skin, enables to stable measurement of biological signals, and obtains high conductivity and durability in comparison with the materials that are applied over clothing. Using an inner shirt type wearable sensor allows a stable collection of biological signals under conditions similar to ordinal conditions without being aware of the measurement. Biometric information is transferred to a smartphone through the transmitter attached to the wearable using Hitoe material. The smartphone displays the biological information transferred by the application implemented using the transmitter software developer's kit (SDK).

Hitoe calculates and displays the stress conditions of the measured subject using LF/HF criteria, but the calculated values are indifferent in our empirical measurements. We believe that frequency domain analysis does not work well in Hitoe application.

\subsection{Poincare Plot}

Poincare plot is another approach to analyze the stress conditions using RRI fluctuation. It marks a plot for a pair of two consecutive RRIs in a two-dimensional (2D) graph. Figure 1 shows a sample of the Poincare plot where the horizontal axis is the RRI value of the $k^{\text {th }}$ cycle in heart beats, shown as RRI $(k)$, and vertical axis is RRI $(k+1)^{(4)}$. The plots are distributed in an elliptical shape around the proportional line $y=x$.

One of the quantitative evaluations of the Poincare plot analyzes the distribution of the ellipse-shaped plots. The RRI values generally become smaller and more stable when we feel nervous and stressed. On the contrary, the values become larger and more dispersed when we feel relaxed. Resultantly, the ellipse area becomes smaller and larger in case of feeling stressed and relaxed, respectively.

\subsection{Formulation of the Poincare Plot}

Toyofuku $^{(3)}$ formulated a new evaluation index based on the size of the ellipse area in the Poincare plot. It defines RRI $(k)$ and RRI $(k+1)$ as a plot in the $\mathrm{x}$ and y-axis, respectively. The ellipse-shaped plots have a long and short axes along a line $y=x$ and $y=-x$, respectively. The deviations for the long and short axes are calculated as follows: (1) the plotted points are projected on $y=x$ and $y=$ $-x$ axes; (2) $\sigma_{\mathrm{x}}$ and $\sigma_{\mathrm{y}}$ is the standard deviation of the distance from the origin on the $y=x$ axis and $y=-x$ axis, respectively, where $\sigma_{\mathrm{x}}$ and $\sigma_{\mathrm{y}}$ are calculated as follows:

$$
\begin{aligned}
& \sigma_{x}=\sqrt{\frac{x^{2}+y^{2}+2 x y}{2}} \text { and } \\
& \sigma_{y}=\sqrt{\frac{x^{2}+y^{2}-2 x y}{2}} .
\end{aligned}
$$

The ellipse area $\mathrm{S}$ of the Poincare plot is calculated as the multiplication of $\sigma_{x}, \sigma_{y}$, and $\pi$, as shown in Eq. (3).

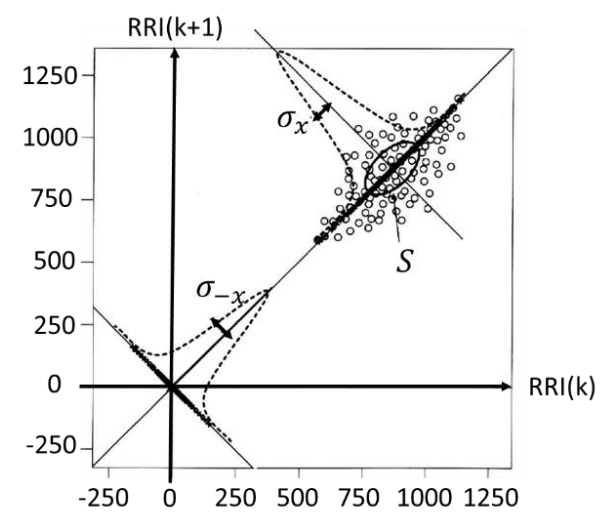

Fig. 1. Poincaré plot graph ${ }^{(4)}$. 


$$
S=\sigma_{x} \times \sigma_{y} \times \pi .
$$

$\mathrm{S}$ is an index for stress since it becomes smaller and larger in case of feeling stressed and relaxed, respectively. The fundamental issue is that even when a person feels relaxed, $\mathrm{S}$ becomes smaller if the subject's heart rate is higher. $\mathrm{S}$ is dependent on the RRI level of the subject. If the subject performs heavy exercise without stress, the subject is determined to be in highly stressed conditions because the size of $\mathrm{S}$ is small.

HRV is sensitive to the autonomic nervous activity. Hence, the traditional methods for analyzing the Poincare plot depend on individual medical differences. Therefore, it is necessary to make a comprehensive judgment by experts with additional indicators. Matsumoto ${ }^{(4)}$ showed a method to analyze the Poincare plot for determining stress conditions semiautomatically. In his analysis, the Poincare plot is generated RRI $(k)$ versus RRI $(k+n)$. Matsumoto ${ }^{(4)}$ showed that the Poincare plot became stable enough to determine the stress conditions if suitable $n$ was selected. $\mathrm{Bu}^{(5)}$ proposed an index, which is applied Poincare plot analysis and developed a distance measure represented inter point relationship, and indicated that the proposed index could provide changes of the stress levels in the short-term duration of pulse rate data.

\section{Quantitative Index of Stress}

RRI values tend to decrease in the case of physical stress during exercise, whereas they tend to increase during relaxation. Therefore, the stress conditions of the RRI values are always judged higher during exercise. On the contrary, their stress conditions might be judged lower even when mental stress is higher but they are not performing an exercise. Stress index should be more robust to reduce the

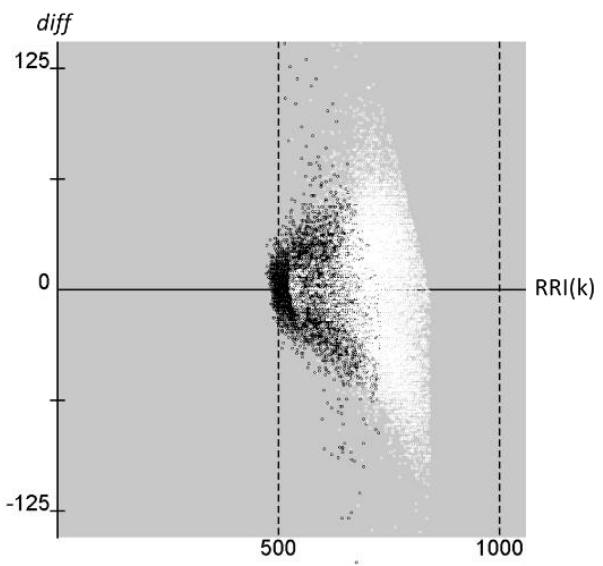

Fig. 2. Graph of RRI changes. effects in the absolute level of RRI. Thus, this paper uses the relative difference between RRI $(k)$ and RRI $(k+1)$ as a stress index because the changes in the RRI values characterize the stress conditions. The RRI changes showing the fluctuation of the electrocardiogram is defined as follows:

$$
\text { diff }=R R I(k+1)-R R I(k) .
$$

Figure 2 shows the changes in the RRI values on a 2D orthogonal graph. The graph is colored black and white when stress is applied and not applied, respectively. Results show that the changes in the RRI values tend to be larger and smaller when absolute RRI levels are larger and smaller, respectively. The stress condition is evaluated as smaller because RRI changes are larger even when stress is heavy. Individual differences exist in the absolute RRI levels in relaxed times, which could be misidentified as being stressed at all times if the RRI values are small even in relaxed times. Thus, the indicator of RRI changes seems significantly affected by the absolute RRI levels.

This study defines a new stress index as the standard deviation of the fluctuation divided by the average RRI value to reduce the influence of the RRI levels. First, when the number of data in a given time is $n$, the average values and standard deviations of the fluctuation for each given period are calculated as follows:

$$
\begin{aligned}
& \mu_{\text {diff }}=\frac{\sum_{k=1}^{n-1} \text { diff }}{n} \text { and } \\
& \sigma=\sqrt{\frac{\sum_{k=1}^{n-1} d i f f^{2}}{n}-\mu_{\text {diff }}^{2} .}
\end{aligned}
$$

The proposed stress index, expressed as $\sigma^{\prime}$, which is the standard deviation relative to the average of the RRI values, can be obtained as follows:

$$
\sigma^{\prime}=\frac{\sigma}{\mu_{\text {diff }}} .
$$

Herein, stress values from 600 data were calculated at 10-min intervals in the experiments. The average $\mu_{\text {diff }}$ and standard deviation $\sigma$ of the RRI values in the sample data were calculated and removed from the RRI values that do not satisfy the following condition to remove noise from the raw biological information.

$$
R R I(k) \leq \mu \pm 2 \sigma .
$$

\section{Stress Load Experiments}

\subsection{Sensor Setting}

The experiments use a wearable sensor with a functional $\mathrm{Hitoe}^{(2)}$ material to measure RRIs. Although 
Hitoe calculates the stress value based on LF/HF theory, the accuracy of its calculation is questionable. Thus, we use Hitoe as a device for measuring electrocardiogram but not as a stress estimator.

\subsection{Evaluation Criteria}

Herein, $t$-test was conducted in a manner similar to the related work ${ }^{(4)}$ to confirm whether a difference exists in the proposed stress indexes for stress and relaxing conditions. Herein, Welch's $t$-test is used to determine the difference between stress and relax samples. Moreover, a single regression analysis is executed and related work method is compared with the coefficient of regression and coefficient of determination to confirm the influence of RRI on stress index.

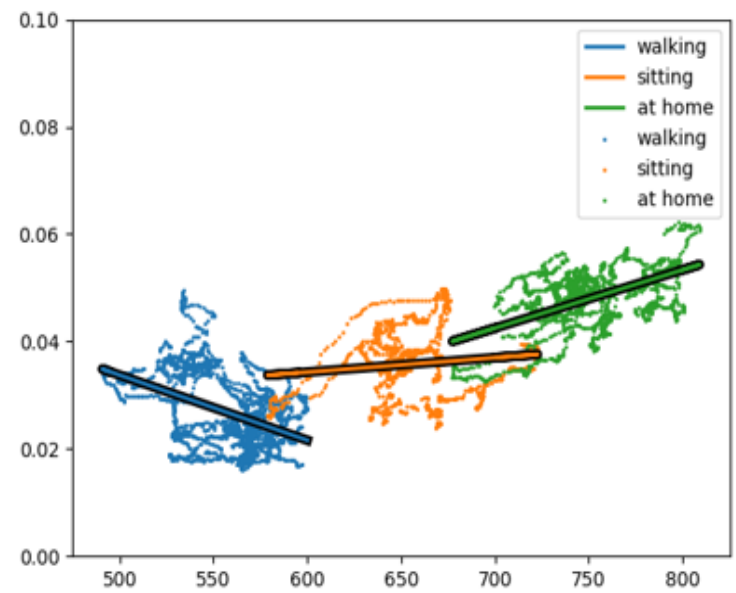

Fig. 3. Stress values and regression lines for physical stress.

Table 1. Stress values statistics for physical stress.

\begin{tabular}{|c|c|c|}
\hline & Proposed method & Related work method \\
\hline between (1) and (2) & $3.87 \times 10$ & $7.00 \times 10$ \\
\hline between (2) and (3) & $1.10 \times 10^{2}$ & $1.82 \times 10^{2}$ \\
\hline between (3) and (1) & $5.41 \times 10$ & $9.87 \times 10$ \\
\hline
\end{tabular}

Table 2. Regression coefficient for physical stress.

\begin{tabular}{|l|c|c|}
\hline & Proposed method & Related work method \\
\hline Situation (1) & $-1.21 \times 10^{-4}$ & $-2.14 \times 10$ \\
\hline Situation (2) & $2.69 \times 10^{-5}$ & $1.26 \times 10^{2}$ \\
\hline Situation (3) & $1.08 \times 10^{-4}$ & $3.39 \times 10^{2}$ \\
\hline
\end{tabular}

Table 3. Determination coefficient for physical stress.

\begin{tabular}{|l|c|c|}
\hline & Proposed method & Related work method \\
\hline Situation (1) & 0.17 & 0.01 \\
\hline Situation (2) & 0.01 & 0.24 \\
\hline Situation (3) & 0.26 & 0.60 \\
\hline
\end{tabular}

\subsection{Physical Stress Experiment}

The subject of this experiment was a 22-year-old female. RRI values were measured in the following three situations: (1) walking outdoors; (2) sitting outdoors; and (3) sitting indoors. Stress values were calculated at 10-min intervals in these three types of situations. Figure 3 shows the calculated stress values and the regression line on the graph. The points of the stress values calculated from situation (1) are blue, those from situation (2) are orange, and the points calculated from situation (3) are green. Table 1 lists the stress values statistics of the proposed and the related work methods. Table 2 shows the results of the regression coefficient of the proposed and related work methods, and Table 3 shows the results of determination coefficient of the proposed and related work methods.

The standard deviations of the proposed stress indexes were smaller when physical stress was larger. The significance of the proposed stress indexes was accepted as $\mathrm{p}<0.005$ by $\mathrm{t}$-test between all the three types of situations when physical stress was applied. This result proved that the presence or absence of stress can be determined by the proposed stress index. A difference exists between situations (2) and (3), and then the physical stress might be accumulated in the measurement just by staying outdoors even if there is no current physical stress due to walking.

\subsection{Mental Stress Experiment}

The subject of this experiment was also a 22 year-old female, which is the same subject in Section 4.3. The changes in the stress index values were examined when the RRI values do not change from the relaxed state. Therefore, the RRI values were measured in the following two situations: (1) sitting and working and (2) sitting and not working. Stress values were calculated at 10 -min intervals in these two types of situations. Figure 4 shows the results of the calculated stress values and the regression line on the graph. The points of the stress values calculated from situation (1) are blue, and those from situation (2) are orange. Table 4 lists the stress values statistics of the proposed and related work methods. Table 5 lists the results of the regression coefficient of the proposed and related work methods, and Table 6 lists the results of the determination coefficient of the proposed and related work methods. 


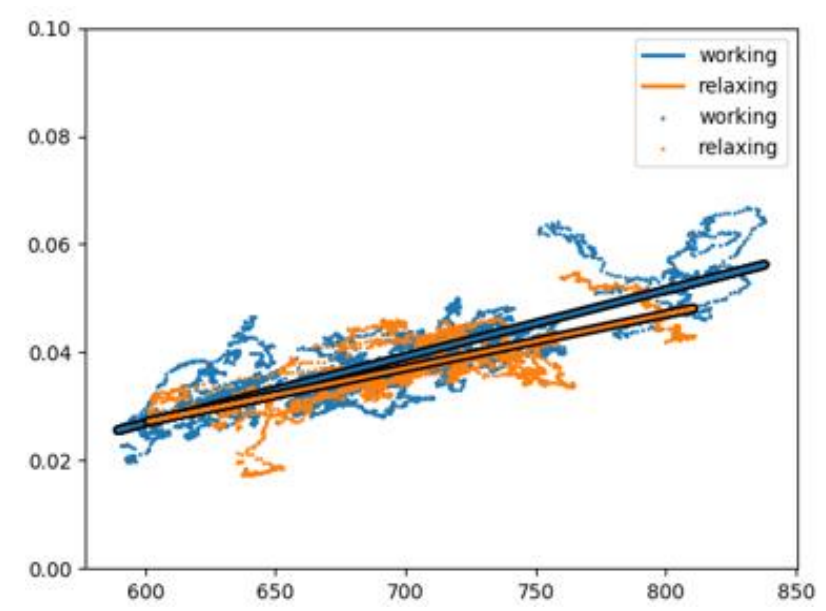

Fig. 4. Stress values and regression lines for mental

Table 4. Stress values statistics for mental stress.

\begin{tabular}{|c|c|c|}
\hline & Proposed method & Related work method \\
\hline Between (1) and (2) & 2.66 & 2.53 \\
\hline
\end{tabular}

Table 5. Regression coefficient for mental stress.

\begin{tabular}{|l|c|c|}
\hline & Proposed method & Related work method \\
\hline Situation (1) & $1.23 \times 10^{-4}$ & $3.18 \times 10^{2}$ \\
\hline Situation (2) & $9.99 \times 10^{-5}$ & $2.77 \times 10^{2}$ \\
\hline
\end{tabular}

Table 6. Determination coefficient for mental stress.

\begin{tabular}{|l|c|c|}
\hline & Proposed method & Related work method \\
\hline Situation (1) & 0.71 & 0.89 \\
\hline Situation (2) & 0.53 & 0.84 \\
\hline
\end{tabular}

The significance of the proposed stress indexes was accepted as $p<0.005$ by t-test between two situations when mental stress was applied.

\section{Discussion}

The regression coefficient values in the three situations in Section 4.3 were smaller than those of the related work method. The proposed stress index succeeded in significantly reducing the influence of the RRI values when physical stress was applied. However, the values of determination coefficients of the three situations were smaller than those in the related work method. The values of the proposed method were widely dispersed and did not converge to constant values. This point requires improvement.

The regression coefficients in the two situations in Section 4.4 were smaller than those of the related work method. Therefore, the influence of the RRI values was reduced in comparison with the stress index in the related work when mental stress was applied. Moreover, the value of determination coefficient in the two situations is larger than the values of the experiment in Section 4.3. The stress values could be converged better than those in the case when physical stress was applied. However, the stress values in situation (2) were smaller than the stress values in situation (1) in both the related work index and the proposed index. Moreover, the changes in the RRI values were smaller when mental stress was applied. Although the influence of the RRI values can be reduced, both the related work stress index and proposed stress index could not distinguish the existence of stress. It might be necessary to examine another judgment material to determine mental stress in addition to the RRI values.

\section{Conclusion}

This study utilized RRI values as the indexes to evaluate the stress conditions by analyzing the autonomic nervous system. The geometrical analysis of HRV in the time domain and new stress index were also proposed. This stress index was able to reduce the influence of the RRI values in comparison with the related work index using the Poincare plot. The stress index performed better than the related work indexes when physical stress was applied. However, it might be necessary to add another judgment material to determine the presence or absence of stress when mental stress was applied.

\section{Acknowledgment}

This work was supported by JSPS KAKENHI Grant Number JP17K00138.

\section{References}

(1) Vesna Vuksanović, Vera Gal : "Heart rate variability in mental stress aloud", Medical Engineering \& Physics, Vol. 29, No. 3, pp. 344-349, 2007

(2) Kazuhiko Takagahara, Kazuyoshi Ono, Naoki Oda, and Takashi Teshigawara : "A wearable sensor that results across industry boundaries - hitoe technology(in Japanese)", Journal of the Society of Life Support Engineering, Vol. 26, No. 5, pp. 42-44, 2014

(3) Fumi Toyofuku, Kazuhiko Yamaguchi, and Hiroshi Hagiwara : "Simplified method for estimating parasympathetic nervous activity by Lorenz plot of 
ECG RR intervals(in Japanese)", Journal of Japan Ergonomics, Vol. 43, No. 4, pp. 185-192, 2007

(4) Yoshiaki Matsumoto, Nobuaki Mori, Ryoh Mitajiri, and Zhongwei Jiang : "Study of Mental Stress Evaluation based on analysis of Heart Rate Variability(in Japanese)", Journal of the Society of Life Support Engineering, Vol. 22, No. 3, pp. 19-25, 2010

(5) Nan Bu : "Stress Evaluation Index based on Poincare Plot for Wearable Health Devices", 2017 IEEE 19th International Conference on e-Health Networking, Applications and Services (Healthcom), pp. 1-6, 2017 